\title{
Imaging very high energy gamma-ray telescopes
}

\author{
Heinrich J. Völk • Konrad Bernlöhr
}

Received: 28 November 2008 / Accepted: 4 February 2009 / Published online: 10 March 2009

(C) The Author(s) 2009. This article is published with open access at Springerlink.com

\begin{abstract}
The technique of $\gamma$-ray astronomy at very high energies (VHE:> $100 \mathrm{GeV}$ ) with ground-based imaging atmospheric Cherenkov telescopes is described, the H.E.S.S. array in Namibia serving as example. Mainly a discussion of the physical principles of the atmospheric Cherenkov technique is given, emphasizing its rapid development during the last decade. The present status is illustrated by two examples: the spectral and morphological characterization in VHE $\gamma$-rays of a shell-type supernova remnant together with its theoretical interpretation, and the results of a survey of the Galactic Plane that shows a large variety of non-thermal sources. The final part is devoted to an overview of the ongoing and future instrumental developments.
\end{abstract}

Keywords History and philosophy of astronomy •

Instrumentation: telescopes - Gamma rays: observation •

Techniques: image processing • Radiation mechanisms:

non-thermal $\cdot$ ISM: supernova remnants

PACS $07.05 . \mathrm{Tp} \cdot 95.55 . \mathrm{Ka} \cdot 98.58 \mathrm{Mj} \cdot 98.70 . \mathrm{Rz} \cdot 98.70 . \mathrm{Sa}$

H. J. Völk (凶) · K. Bernlöhr

Max-Planck-Institut für Kernphysik, P.O. Box 103980, 69029 Heidelberg, Germany

e-mail: Heinrich.Voelk@mpi-hd.mpg.de

K. Bernlöhr

Institut für Physik, Humboldt-Universität zu Berlin, Berlin, Germany

e-mail: Konrad.Bernloehr@mpi-hd.mpg.de 


\section{Introduction}

At $\gamma$-ray energies $E_{\gamma} \gtrsim 30 \mathrm{MeV}$ electron-positron pair production in the Coulomb field of an atomic nucleus dominates the interactions of photons with normal matter. Cosmic $\gamma$-rays of such energies can therefore be most effectively measured in either gas-filled or solid state satellite detectors above the atmosphere registering the tracks of the resulting $e^{ \pm}$-pairs, or using the Earth's atmosphere itself as detector in observing secondary Cherenkov radiation with ground-based telescopes. Satellite detectors work from $\sim 30 \mathrm{MeV}$ up to some tens of $\mathrm{GeV}$, where they become statistics-limited as a result of the limited size of detectors that can be transported by rockets into space. In the Very High Energy range (VHE; $E_{\gamma}>100 \mathrm{GeV}$ ) large optical telescopes are used, often with diameters of $10 \mathrm{~m}$ or more.

The history, development and results of satellite $\gamma$-ray detectors have been described in this volume by K. Pinkau. Addressing the complementary ground-based technique, we shall in the following summarize the physics of UV/Optical imaging atmospheric Cherenkov telescopes which register the Cherenkov radiation from the atmospheric secondary particles. Subsequently we shall discuss two important astrophysical results that demonstrate the power of this observation method. Given our personal involvement in the H.E.S.S. (High Energy Stereoscopic System) array in Namibia we shall in most instances use this experiment as example (see Fig. 1), although we shall also emphasize the other large arrays existing worldwide. The scientific results are astonishing, given the slow development of the field over the three decades before the mid-1990s. We conclude with a glimpse at the next generation European project that envisages a further sensitivity increase by an order of magnitude.

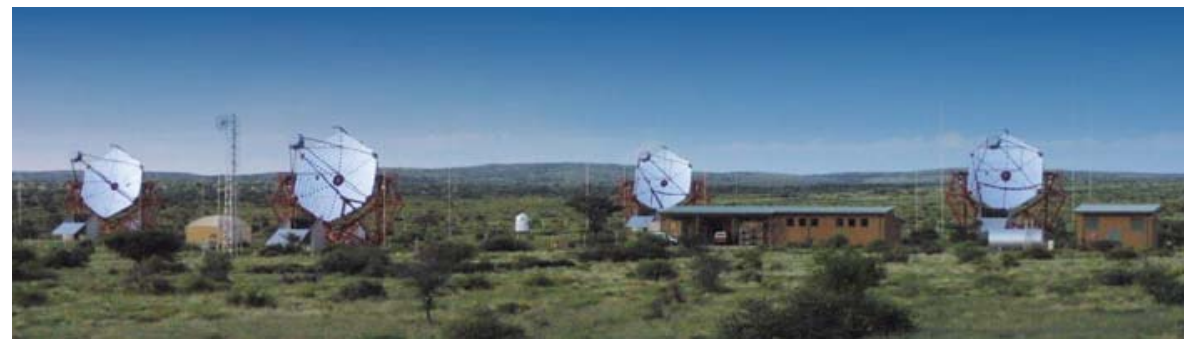

Fig. 1 The H.E.S.S. telescope array [1] in the Khomas Highland of Namibia at an elevation of $1800 \mathrm{~m}$ a.s.l. The four $13 \mathrm{~m}$ telescopes stand at the corners of a square with a side length of $120 \mathrm{~m}$ 


\section{Air showers, secondary atmospheric Cherenkov emission, imaging atmospheric Cherenkov telescopes}

High-energy cosmic gamma rays produce $e^{ \pm}$pairs in the atmosphere over ${ }_{7}^{9} X_{0}$ (with the radiation length $X_{0} \approx 36.5 \mathrm{~g} / \mathrm{cm}^{2}$ in air). This is followed by Bremsstrahlung over the next radiation length which implies new $\gamma$-rays that generate new pairs, etc., until the energy of the final generation of electrons becomes so small that their fate is dominated by ionization losses which rapidly cool and thermalize them. The result of these processes is called an electromagnetic Air Shower that exists for about $10^{-4} \mathrm{~s}$ while traversing the atmosphere. The multiplication of the number of particles and their eventual removal by thermalization leads to a maximum number of shower particles at about 250 to $450 \mathrm{~g} / \mathrm{cm}^{2}$ for primary $\gamma$-rays of $20 \mathrm{GeV}$ to $20 \mathrm{TeV}$, corresponding to an atmospheric height of about 7 to $12 \mathrm{~km}$ above the ground.

The most realistic and complete description of the physical processes and the corresponding results in the detector(s) is given by Monte Carlo (MC) simulations. Except when explicitly noted we use the simulations performed by one of us [2] which use the CORSIKA code [3]. It incorporates all the physics of the atmospheric interactions and emission processes and gives the possibility to statistically track their evolution in the atmosphere, down into the detector.

Not only $\gamma$-rays penetrate into the atmosphere, but also charged energetic nuclei (Cosmic Rays). They must be distinguished from the $\gamma$-rays. Energetic protons and nuclei undergo hadronic interactions and produce dominantly neutral $\left(\pi^{0}\right)$ or charged $\left(\pi^{ \pm}\right)$pions. Whereas the former decay into two gammas, the latter ultimately produce electrons, positrons and two neutrinos via the $\pi \rightarrow \mu \rightarrow e$ decay. This leads to background air showers which are of a mixed hadronic and electromagnetic nature. As a result of the large transverse momentum transfer in hadronic interactions the hadronic shower component is broad and irregular compared to the electromagnetic component (see Fig. 2).

Fig. 2 The different

character of gamma showers

and hadronic showers. The

gamma shower is slender and

to lowest approximation

axially symmetric about the

direction of the primary.

The hadronic shower is irregular and may contain electromagnetic subshowers as a result of the large transverse momenta generated in hadronic interactions (from K. Bernlöhr)

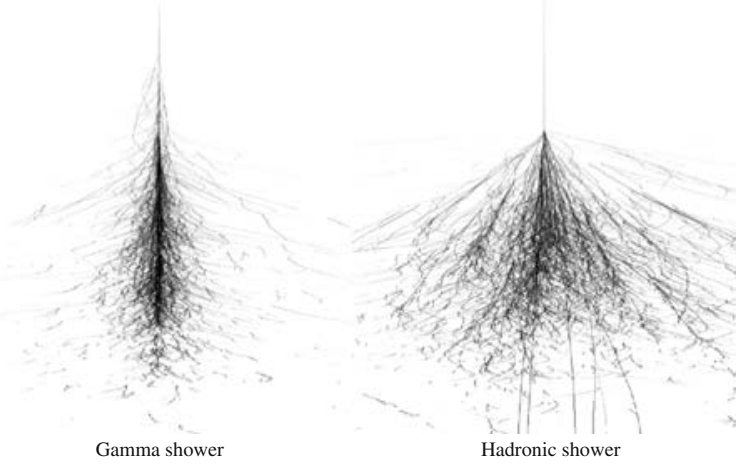


In general the flux of Cosmic Ray (CR) particles is much larger-by a factor of about $10^{3}$-than that of $\gamma$-rays. This implies a large background for $\gamma$ ray astronomy from the ground. It must be separated from the $\gamma$-ray signal, because no anti-coincidence shield can be applied as in space detectors.

At primary $\gamma$-ray energies of about $100 \mathrm{GeV}$ very few energetic photons or electrons reach the ground. But the shower electrons from the original $\gamma$-ray are still observable with optical telescopes through their Cherenkov radiation in the optical range, because this atmospheric Cherenkov emission reaches the ground without major absorption. Figure 4 below gives an impression of the overall configuration. The disadvantages of this very promising measurement technique are the weakness of the Cherenkov light and, to some extent, its optical character. They require large light collection devices and limit the observation time to clear and moonless nights. The observation efficiency is typically about $10 \%$, depending critically on the astronomical quality of the site.

\subsection{Cherenkov light pool}

The atmospheric Cherenkov light emission from a single particle is characterized by a forward cone with an opening angle $\Theta \approx 1^{\circ}$ that increases downwards. For a particle moving vertically downwards, the largest ring on the ground near sea level is from a height of 12 to $15 \mathrm{~km}$ (see Fig. 3).

The ensemble of shower electrons from an energetic primary $\gamma$-ray produces a rather uniformly illuminated "light pool" on the ground, centered on the shower core, with a radius of about $125 \mathrm{~m}$, if the multiple scattering of the
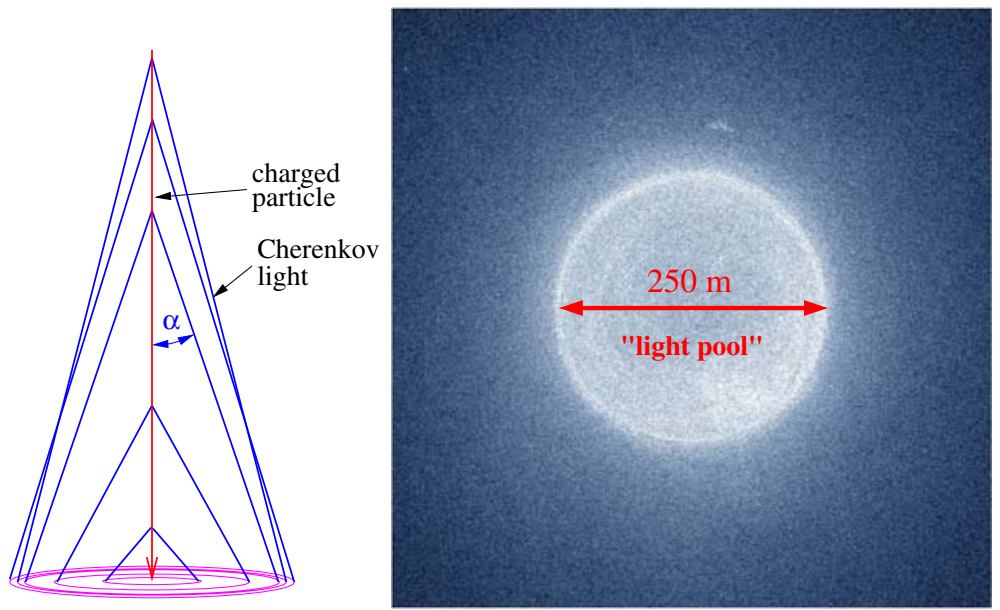

Fig. 3 Left: Atmospheric Cherenkov emission from a downward-moving single particle. Right: The "light pool" at an observation level at $1800 \mathrm{~m}$ above sea level from a $\gamma$-ray shower with a primary energy of $1 \mathrm{TeV}$ (from K. Bernlöhr) 
shower electrons is included (see Fig. 3). To first approximation it corresponds to the effective area of a telescope that images the shower.

A schematic picture of a shower from a cosmic $\gamma$-ray source, illuminating an array of telescopes on the ground, is given in Fig. 4. Since the atmospheric index of refraction is very close to 1 , the Cherenkov light almost keeps pace with the radiating charged particles. Near the edge of the "light pool" most light from a $\gamma$-ray shower arrives within 2 ns (see Fig. 5). Thus a very short temporal window is possible, in order to suppress the dominant night sky background. This suggests the use of fast photomultiplier cameras for the telescopes.

\subsection{Atmospheric Cherenkov telescopes}

The lateral distribution of the Cherenkov photon density depends somewhat on the atmospheric profiles in its amplitude. However, the dependence on the distance from the shower core-the extrapolation of the direction of the primary $\gamma$-ray-is essentially independent of the atmospheric conditions. An example is shown in Fig. 5. At a $\gamma$-ray energy of $100 \mathrm{GeV}$ about 1000 Cherenkov photons are produced in a $100 \mathrm{~m}^{2}$ telescope. With a conversion efficiency of $10 \%$ this results in $\sim 100$ photoelectrons in the image. Given that the total number of Cherenkov photons is about proportional to the primary $\gamma$-ray energy, this determines the threshold energy of the telescope.

Fig. 4 Schematic of the Cherenkov light pool, originating from a primary $\gamma$-ray from within a cosmic-ray source (e.g. a supernova remnant) that illuminates an array of telescopes (from K. Bernlöhr)

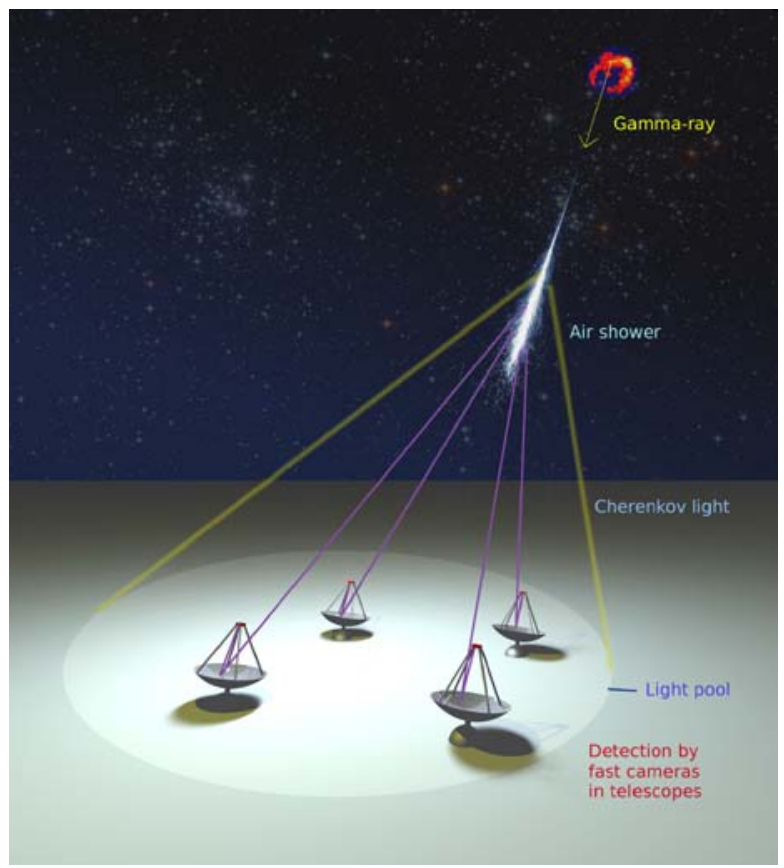



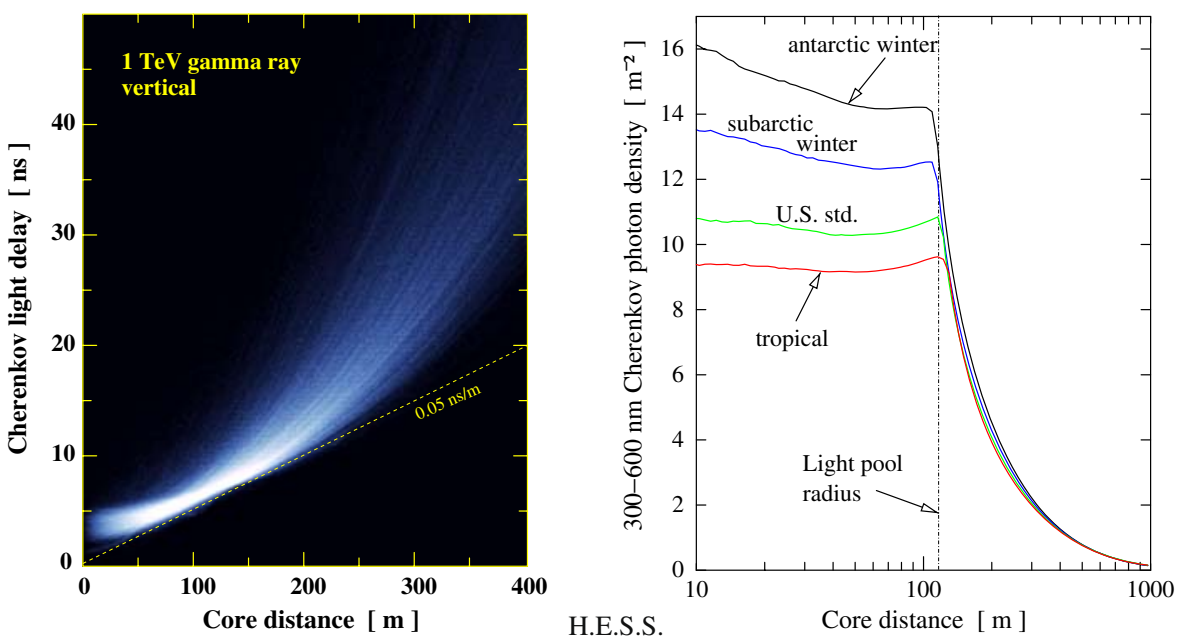

Fig. 5 Left: Distribution of arrival time and core distance of Cherenkov photons in a $1 \mathrm{TeV}$ $\gamma$-ray-shower. Time is counted with respect to the time of arrival of the primary $\gamma$-ray on the ground assuming no interactions. Right: Lateral distribution per unit area of the optical Cherenkov emission from a shower with primary energy of $100 \mathrm{GeV}$, for various atmospheric profiles (from K. Bernlöhr)

After successful initial experiments concerning CR air showers-what we call today the CR background-in the 50ies in the UK [4] and in the USSR [5], the first dedicated $\gamma$-ray observations were attempted in the Crimea by the Lebedev group from Moscow, following the suggestion by [6]. At the time the results were basically negative [7]. Nevertheless they resulted in an upper limit to the VHE $\gamma$-ray flux from the Crab Nebula. ${ }^{1,2}$

In the USA the growing interest in VHE $\gamma$-ray astronomy led to the construction (in 1968) of a $10 \mathrm{~m}$ optical reflector with tessellated mirrors at the Whipple Observatory in southern Arizona (see Fig. 6). ${ }^{3}$ The imaging of the shower's Cherenkov light is schematically shown in Fig. 6.

Despite intriguing indications the results remained controversial for about 20 years. With a multi-pixel photomultiplier camera in the focus since the mid80ies and the introduction of image analysis finally the Crab Nebula could be

\footnotetext{
${ }^{1}$ A very interesting account of the early efforts in the USSR is given by A.S. Lidvansky in the talk "Air Cherenkov Methods in Cosmic Rays: A Review and Some History" presented at the centenary conference "P.A. Cherenkov and Modern Physics" (Moscow, 2004) [8].

${ }^{2}$ A condensed history of the field until 1994, very worthwhile reading, is to be found in [9].

${ }^{3}$ Of the scientists originally involved (G.G. Fazio, J.E. Grindlay, G.H. Rieke, T.C. Weekes, and others) several have later also become leaders in fields like Infrared Astronomy and X-ray Astronomy.
} 

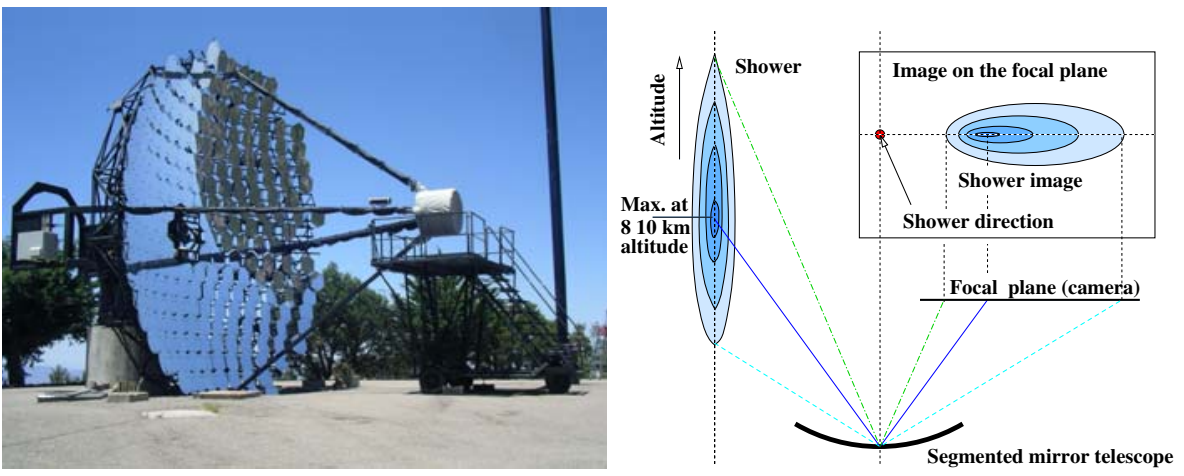

Fig. 6 Left: The $10 \mathrm{~m}$ Whipple telescope on Mt. Hopkins, USA [11]. Right: Shower imaging by a telescope. Despite some asymmetry, the shower image in the camera has the shape of an ellipse. The shower direction is a point somewhere on the extension of its major axis. For $\gamma$-ray primaries the image intensity gives the primary energy (from K. Bernlöhr)

detected with high $(9 \sigma)$ significance after $60 \mathrm{~h}$ of observation time [10]. Even after this breakthrough and a number of further significant detections, the field continued to evolve rather slowly until the mid-90s when a number of new telescopes started operation. They introduced the stereoscopic technique and very fine camera pixelation (see below), leading up to the present group of four major telescope systems worldwide.

The difficulties in the early $\gamma$-ray observations may be traced back to the massive background of nuclear CR events. In addition, the large spatial extension of air showers in the direction of the primary photon trajectory leads to a very extended image $\sim 1^{\circ}$ in the camera plane (see Fig. 7), making the size of the field of view (FoV) of the camera a critically important parameter of the system. The lateral spread and irregularity of hadronic showers increases this extension into the second dimension and makes itself visible in the camera through an irregularly structured image.

This difference between $\gamma$-rayshowers and hadron showers can be used to distinguish the nature of the generating particles through image analysis, given a sufficiently dense array of photomultipliers in the camera [12].

The aim of Hillas' algorithm was to reject the dominant events from CR nuclei by suitable cuts on the images that are derived from Monte Carlo simulations. The application of this method has proven to be very successful. The remaining problem is that with a single telescope one obtains only one projection of the Cherenkov light "shower". In addition, a single telescope suffers from a second kind of background effect. It is produced by penetrating muons (from CR interactions) that reach the ground at the telescope mirror or its immediate neighborhood. These muon events are not easily distinguishable from $\gamma$-rays. The solution for these drawbacks consists in the stereoscopic technique. It was pioneered in the HEGRA telescope array on La Palma [13] that started full operation in the mid-90s (see Fig. 8). 


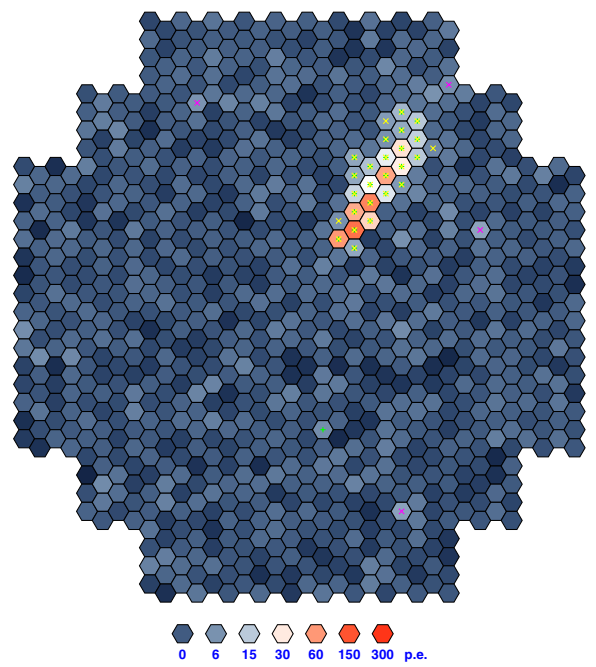

$1.0 \mathrm{TeV}$ gamma shower

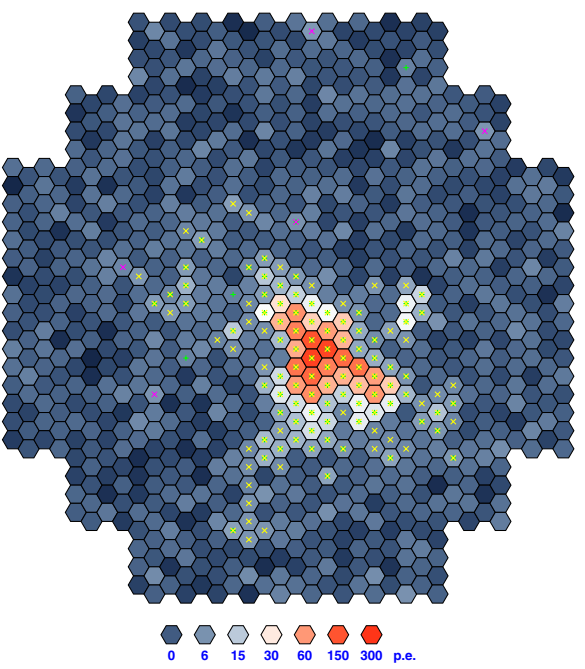

$2.6 \mathrm{TeV}$ proton shower

Fig. 7 Difference between the images of gamma-induced and hadron-induced showers in the camera (from K. Bernlöhr)

\subsection{Stereoscopic method}

The use of several telescopes observing the same shower in coincidence allows a unique determination of the shower direction by projecting the images in all triggered telescope cameras into one camera (see Fig. 9). Then the intersection point of the image major axes yields the shower direction. Compared to a single telescope the angular resolution, the energy resolution, the background rejection and the sensitivity are improved. In addition this method allows the 3-dimensional reconstruction of the shower, including the height of maximum

Fig. 8 The start of stereoscopy: HEGRA on La Palma (1995-2002) [14]. The five $3.5 \mathrm{~m}$ telescopes were situated in the center and at the 4 corners of a square of $100 \mathrm{~m}$ sidelength. The FoV was $\approx 5^{\circ}$

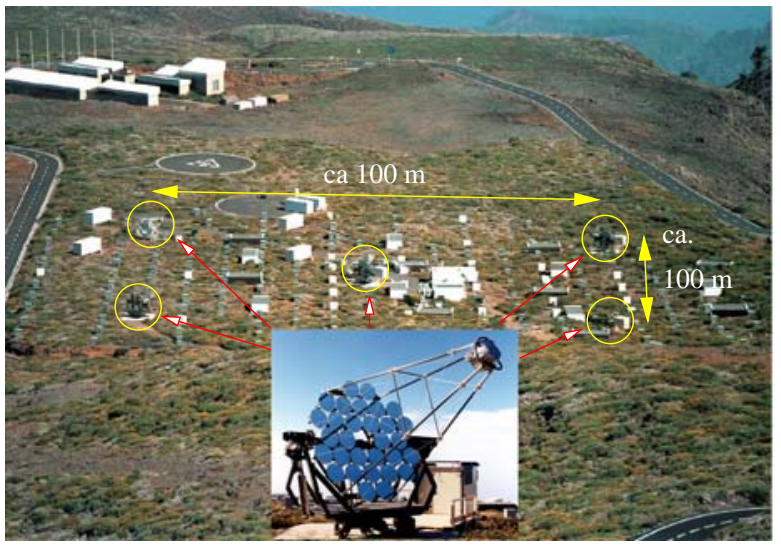




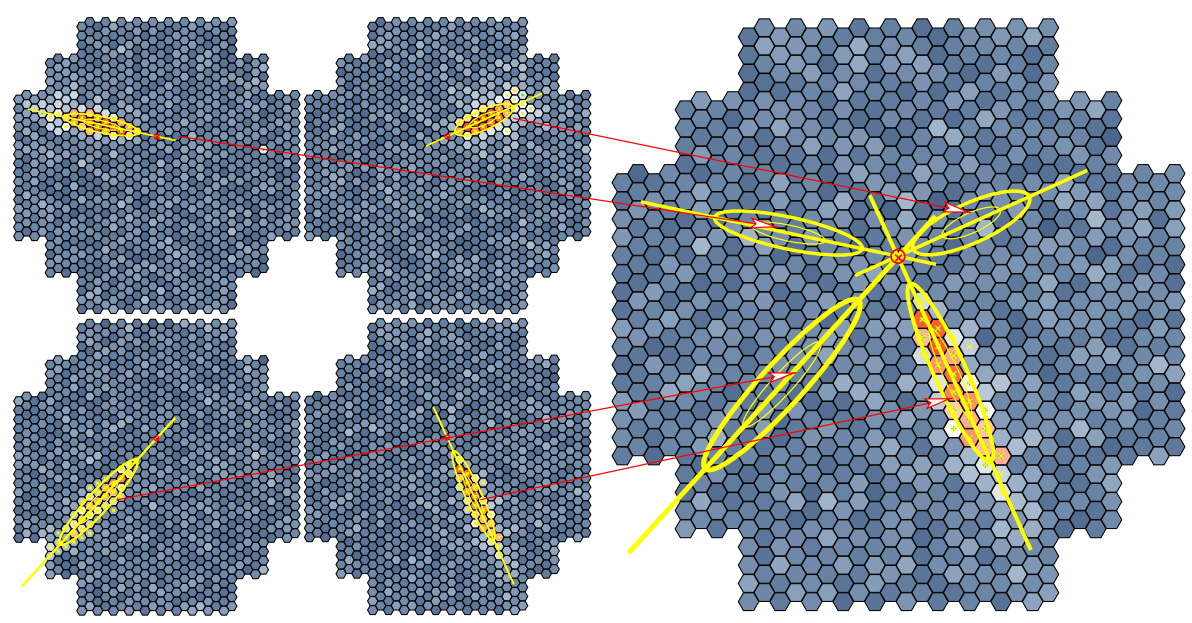

Fig. 9 Projection of several images from within the light pool of an event into one camera plane in a stereo system with four telescopes (from K. Bernlöhr)

particle number. The most advanced data analysis methods use 3-dimensional modeling of the shower, no longer confining themselves to the use of the 2-dimensional image parameters alone.

The second major step forwards in the stereoscopic observation mode is the suppression of the above-mentioned local muons with a stereo trigger: they leave an image only in the telescope concerned, but not in the other telescopes (unless the telescope light-gathering power is so enormous that even a single charged particle can trigger an event from an inter-telescope distance, a case which we will not discuss here). Therefore such events can be almost completely eliminated (see Fig. 10). This is most important near the energy threshold, where the shower images get weak and poorly defined. In other
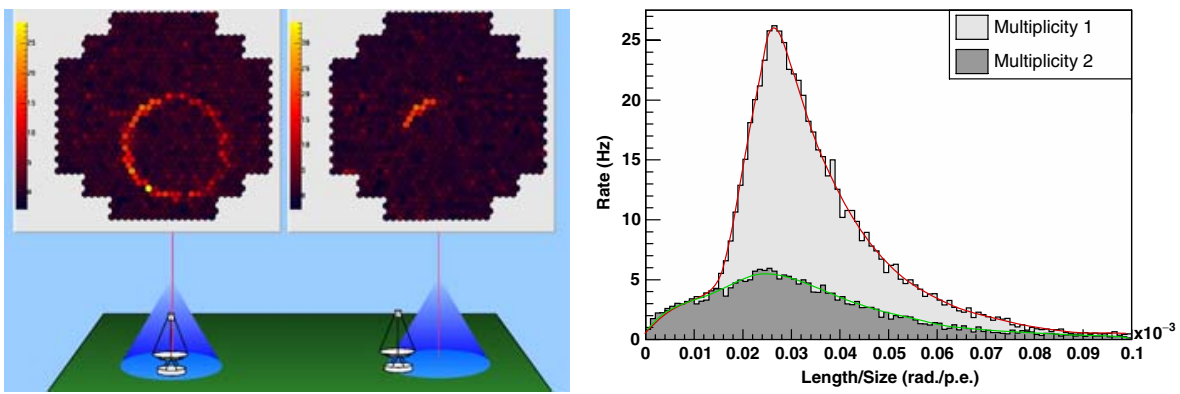

Fig. 10 Local muons hitting telescopes in the center and close to the periphery (left, courtesy G. Hermann and W. Hofmann). In peripheral encounters the resulting image is difficult if not impossible to distinguish from a low-energy $\gamma$-ray shower (right, based on H.E.S.S. data [15]). No such effect occurs in the other telescopes of a multiple system. The exclusion of muon events in stereoscopic systems reduces the muon background drastically 
words, only stereoscopic systems can reach the theoretical energy threshold derived from the mirror size. Single telescopes are severely hampered by the muon background and must operate significantly above their theoretical threshold.

Despite the small mirror sizes of its components, the HEGRA array became the most sensitive VHE instrument of its kind. It could thus prove the promise of stereoscopy. Among others, several Blazar-type extragalactic sources, like the active galactic nuclei Mrk 421 and Mrk 501, discovered shortly before by the Whipple telescope, were confirmed or even measured with improved quality. The $\gamma$-ray emission of the young Galactic Supernova Remnant Cassiopeia A was discovered in a series of deep observations over a period of $232 \mathrm{~h}$, and an unidentified VHE $\gamma$-ray source in the Cygnus region was detected; its astrophysical origin is subject of discussion to this day. In spite of these important results the HEGRA array was not large enough to see more than the "eight-thousanders" of the VHE range. Its scan of the Galactic scan revealed no new source and was in this sense not successful. The other existing instruments of the time-the Japanese-Australian CANGAROO I telescope at Woomera and the British Durham Mark 6 telescope in Narrabri, both in the southern hemisphere in Australia, the French CAT telescope in the French Pyrenees (championing very fine camera pixelation), and others, together with the Whipple telescope-all shared this problem (for an overview of these instruments see [16]).

\subsection{Third generation instruments; H.E.S.S.}

As a consequence, a third generation of imaging Cherenkov telescopes was envisioned and eventually realized. These were the VERITAS array [17], planned for the Mt. Hopkins area again, CANGAROO III [18] near Woomera, H.E.S.S. in Namibia [19]—also in the southern Hemisphereand MAGIC [20] on La Palma at the HEGRA site. They all were to have high-definition cameras and, with the exception of MAGIC that was initially designed and operated until recently as a large single telescope, they were stereoscopic arrays, following HEGRA (see Fig. 11).

As an example let us consider H.E.S.S. (High Energy Stereoscopic System $).{ }^{4}$ The experiment's name is at the same time a tribute to Victor F. Hess, the Austrian physicist who discovered the Cosmic Rays in 1912 in a series of carefully instrumented high-altitude balloon flights. He received the Nobel prize for this discovery in 1938.

The H.E.S.S. Collaboration involves a total of about 150 scientists from various European countries, Armenia, South Africa and Namibia. While the astrophysicists play a decisive role, the majority are physicists that adapt techniques from accelerator physics to this young field of astronomy. The

\footnotetext{
${ }^{4}$ The conceptual basis for stereoscopic systems of $\sim 10 \mathrm{~m}$ telescopes is given in [21, 22].
} 
Fig. 11 The third generation imaging Cherenkov telescopes: the Japanese-Australian array CANGAROO III [23] the two European initiatives H.E.S.S. [1] and MAGIC [24], and the US-observatory VERITAS [25]
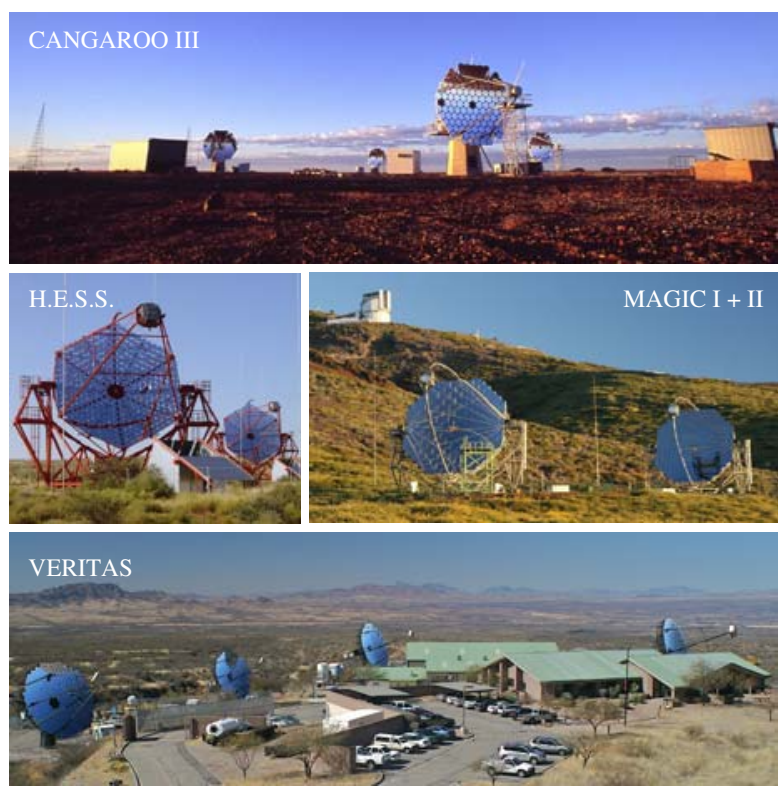

pattern is reminiscent to that visible in the development of radio and X-ray astronomy some decades earlier.

The 4-telescope array (see also Fig. 1) is designed for maximum mechanical stability despite full steering and automatic remote alignment capability of the 382-piece mirror system that implies an area of $107 \mathrm{~m}^{2}$ per telescope; the individual glass mirrors have a diameter of $60 \mathrm{~cm}$. The camera in the primary focus weighs almost 1 ton (see Fig. 12).

As mentioned before, the large field of view of $5^{\circ}$ of the telescope cameras is a key feature of H.E.S.S. Even though quite expensive in cost, operation and

Fig. 12 One of the H.E.S.S. cameras in its shelter [1]. It has 960 photomultiplier pixels of size $0.16^{\circ}$, resulting in a $5^{\circ}$ field of view. The electronics with $1 \mathrm{GHz}$ analog sampling rate is entirely contained in the camera body

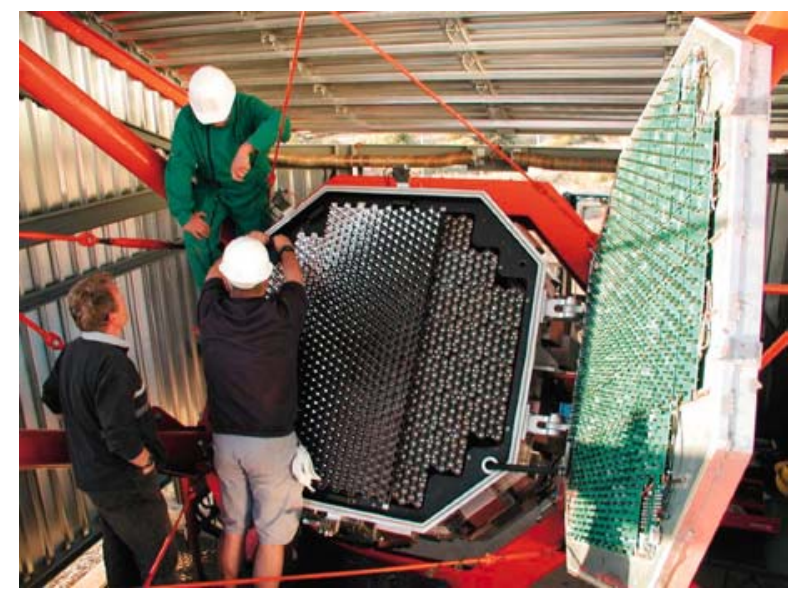



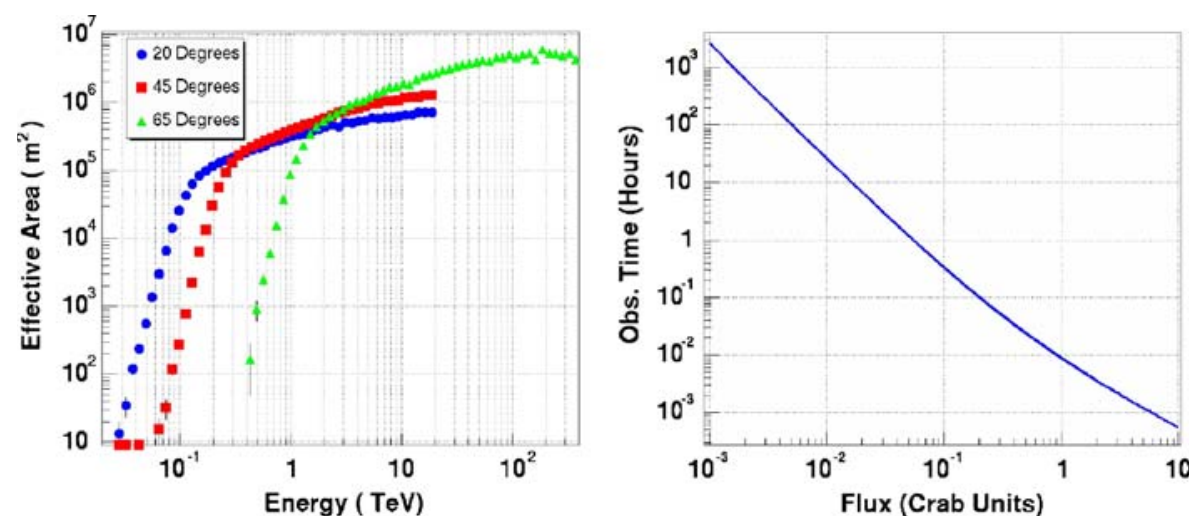

Fig. 13 Performance of the H.E.S.S. 4-telescope system. The effective area as a function of $\gamma$-ray energy is shown (left). The right panel gives the observation time required for a $5 \sigma$ detection as function of the $\gamma$-ray flux in units of the flux from the Crab Nebula $([19,28])$

maintenance, this requirement resulted from the realization that most nearby Galactic VHE sources should be extended by $\sim 1^{\circ}$ degree or more, e.g. [26]. This should be also the case for the VHE emission from nearby rich clusters of galaxies, one of the key targets of extragalactic $\gamma$-ray astronomy [27].

Only a large field of view permits efficient observations of such extended objects. In addition it makes it possible to perform an efficient sky survey, especially in the Galactic Plane. Beyond that, the collection area of the telescopes increases with increasing $\gamma$-ray energy, since the Cherenkov light pool extends beyond $125 \mathrm{~m}$ for very energetic showers that produce a large amount of Cherenkov light. For the H.E.S.S. telescopes this implies a considerable increase of the collection area with energy up to $\gamma$-ray energies of about $100 \mathrm{TeV}$.

The resulting performance of the H.E.S.S. array is summarized in Fig. 13. After suitable data cuts on the expected direction of the source and on the shape of the images, derived from Monte Carlo simulations [28], the analysis accepts more than $50 \%$ of the gamma events and less than $0.1 \%$ of the events from CR nuclei; the angular resolution is better than $0.1^{\circ}$ per event and the energy resolution is $10-15 \%$ per event. This compares favorably with satellite detectors. The energy threshold (at zenith) is $100 \mathrm{GeV}$. The collection area increases with $\gamma$-ray energy, reaching $>1 \mathrm{~km}^{2}$ for observations at large zenith angles. The sensitivity in the overlapping energy range is a factor of $\approx 10$ better than that of HEGRA and exceeds that of the single-telescope MAGIC by a factor $\sim 3$. This implies an observation time of $1 \mathrm{hr}$ for a $5 \sigma$ detection of an energy flux of $10^{-11}$ and $10^{-12} \mathrm{erg} \mathrm{cm}^{-2} \mathrm{~s}^{-1}$ at $\gamma$-ray energies of $100 \mathrm{GeV}$ and $1 \mathrm{TeV}$, respectively. In other words, an instrument like H.E.S.S. can detect the (point-like) Crab Nebula in $\mathrm{TeV} \gamma$-rays in about $30 \mathrm{~s}$, compared with the $\sim 18 \mathrm{~h}$ required for the equivalent original detection 15 years earlier. ${ }^{5}$

\footnotetext{
${ }^{5}$ The Crab Nebula is generally treated as standard candle in VHE $\gamma$-ray astronomy.
} 


\section{Two major scientific results}

In this section we shall illustrate the technique of ground-based $\gamma$-ray astronomy with two examples from the H.E.S.S. results. They share the ubiquitous feature of spatial extension, characteristic of so many of the non-thermal sources in the Universe. The first example is a Supernova Remnant (SNR), thus possibly belonging to the long-sought class of sources of the Galactic CRs. The other example concerns the results of the Galactic Plane scan in the VHE range that has been performed since 2004 .

\subsection{The supernova remnant RX J1713.7-3946}

This SNR, located in the constellation Scorpius in the Galactic plane, was originally discovered with the X-ray satellite ROSAT [29]. In X-rays RX J1713.73946 has a diameter of $1^{\circ}$, twice the size of the full moon (see Fig. 14). The ASCA (1-3 keV) image shows an overall shell structure ([30]). Also in subsequent observations with Chandra and XMM the X-ray emission was "entirely" non-thermal: no thermal emission has yet been identified. The existence of a central X-ray point source, which is probably a neutron star, suggests the core collapse of a massive star. RX J1713.7-3946 was also detected with H.E.S.S. [31, 32] and there exists a close spatial correlation of the X-ray and the VHE $\gamma$-ray emission (see Fig. 14).

The $\gamma$-ray spectrum is very hard-harder than a spectrum containing equal energy per decade in the observed range-and extends up to $\gamma$-ray energies of about $100 \mathrm{TeV}$ [33] (see Fig. 15). This means that the generating charged

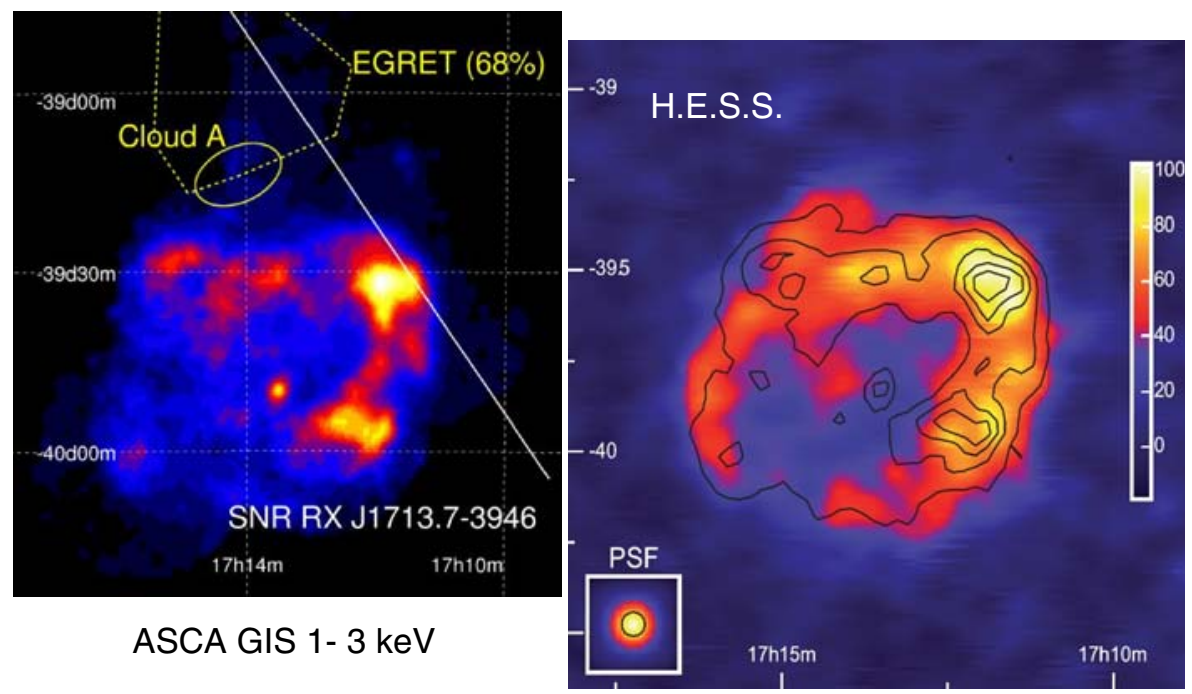

Fig. 14 Left: Image of the Supernova Remnant RX J1713.7-3946, as observed with the ASCA satellite in the 1-3 keV band [30]. Right: H.E.S.S. image of RX J1713.7-3946 at energies above $190 \mathrm{GeV}$ (in false colors), with the ASCA contours superposed [33] 

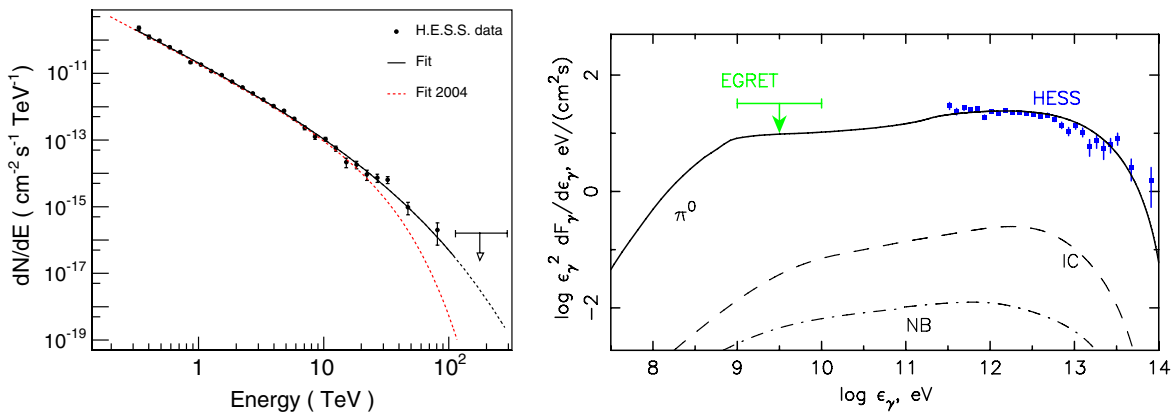

Fig. 15 Left: H.E.S.S. energy spectrum of RX J1713.7-3946 [33]. Right: Theoretical spectral energy distributions of RX J1713.7-3946 in the VHE range, assuming a magnetic field strength $B_{\text {eff }} \approx 130 \mu \mathrm{G}$ inside the remnant [35]

particles must have energies in excess of about $300 \mathrm{TeV}$ and, in the case of nuclear particles, even of about $600 \mathrm{TeV}$. A reasonable phenomenological fit to the differential $\gamma$-ray energy spectrum is $d N / d E=N_{0} E^{-\Gamma} \exp \left(E / E_{\mathrm{c}}\right)^{0.5}$, with $\Gamma=1.8 \pm 0.04$ and $E_{\mathrm{c}}=3.7 \mathrm{TeV}$. Extrapolating this spectrum back to $1 \mathrm{GeV}$, using arguments from diffusive shock acceleration theory (see below), yields a total energy in energetic particles of about $10^{50} /\langle n\rangle$ erg. For an average gas density of $\langle n\rangle \approx 1 \mathrm{~cm}^{-3}$ this corresponds to roughly 10 percent of the expected total hydrodynamic explosion energy. Also spectral imaging and an energyresolved morphological characterization was achieved.

Given the possible fundamental significance of such an empirical finding we show a comparison of the spatially-integrated spectral energy distribution (SED) from H.E.S.S. with a theoretical model spectrum [34, 35] that assumes a magnetic field strength $B_{\text {eff }} \approx 130 \mu \mathrm{G}$ inside the remnant and is able to fit the amplitude of the VHE spectrum (see Fig. 15) for a typical injection rate of supra-thermal nuclear particles into the acceleration process. The inverse Compton (IC) emission and Bremsstrahlung (NB) of the simultaneously accelerated CR electrons turn out to be negligible in comparison with the $\gamma$-ray emission generated by the $\pi^{0}$-production and subsequent decay into two $\gamma$-rays from inelastic collisions of the accelerated nuclear particles with thermal gas nuclei in the interior of the remnant. The upper limit in the GeV range, derived for this region of the sky from the EGRET detector, is shown as well. The IC and NB amplitudes in Fig. 15 are derived from the observed synchrotron SED in the radio and X-ray ranges below $100 \mathrm{keV}$ (see Fig. 16), consistent with the magnetic field strength above.

That 10 or more percent of the entire mechanical explosion energy of the Supernova explosion should be transformed into non-thermal energy of ultrarelativistic particles is a rather outrageous proposition from the theoretical side. Yet nature might indeed comply with it, as the H.E.S.S. result shows. The predicted hadronic dominance of the $\gamma$-ray SED is expected to be confirmed by the recently launched Fermi Gamma-ray Space Telescope with an SED that 
Fig. 16 Overall and spatially-integrated non-thermal spectral energy distribution of

RX J1713.7-3946. The lower-energy synchrotron observations in the radio (ATCA) and X-ray (ASCA and Suzaku) ranges is compared with the theoretical model [35]. The $\gamma$-ray spectrum at higher energies is the same as in Fig. 15

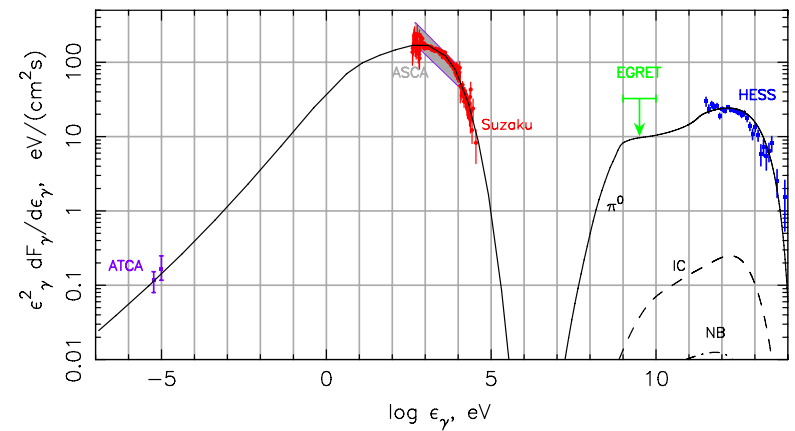

should be only a factor $\approx 2.5$ lower at $1 \mathrm{GeV}$ than that measured at $1 \mathrm{TeV}$ from Fig. 15.

\subsection{Galactic Plane survey}

H.E.S.S. has also performed a survey of the Galactic Plane within a band of $-3<b<3$ degrees in Galactic latitude $b$ (see Fig. 17) with a $3 \mathrm{~h}$ exposure
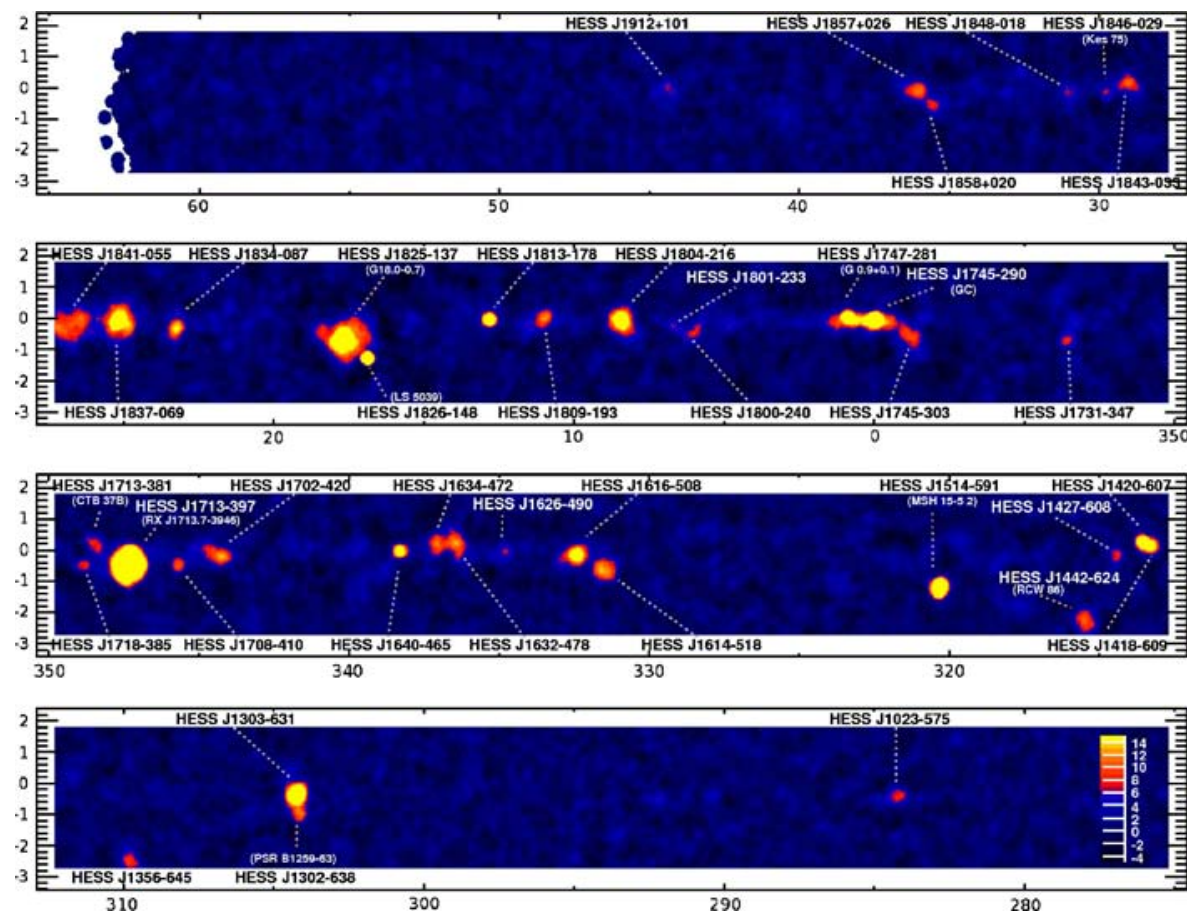

Fig. 17 Significance map of the Galactic Plane Survey by H.E.S.S., presented in Galactic coordinates: $280^{\circ}<l<60^{\circ}$ and $-3^{\circ}<b<3^{\circ}$ [36]. Most of the sources are spatially extended beyond the $0.1^{\circ}$ resolution of the array. The Galactic Center (GC) region and the SNR RX J1713.7-3946 can be seen in the right half of the 2 nd panel and the left half of the 3rd panel from the top, respectively 
per field. The scan resulted in about 40 sources of at least $5 \sigma$ significance (status 2007) in a band $280<l<60$ degrees of Galactic longitude $l$, most of them spatially extended [36]. About half of these sources are unidentified in other wavelength ranges. One gets the impression that the amazing number of sources and variety of source types almost fills the nearby part of the plane, as the significance map Fig. 17 suggests. The source types range from Pulsar Wind Nebulae, Pulsars in binary systems, X-ray Binaries, shell-type SNRs and OB associations to giant molecular clouds and the Galactic Center region. The survey is presently continued. The number of VHE sources, at least in this special part of the sky, appears mainly limited by the sensitivity of the instrument and possibly also by confusion, rather than by the availability of sources.

\section{The future}

The fact that the VHE sky is rich in sources-also in extragalactic space, as recent detections by several observatories have shown - has led to efforts to increase the power of the existing detectors. MAGIC started to experiment with advanced solid state detectors in telescope cameras and to build a second telescope, in order to be able to do stereoscopic observations. This second telescope is currently_fall 2008 - in the commissioning phase (see Fig. 11). H.E.S.S. began in 2006 to build a $28 \mathrm{~m}$ telescope in the center of the original H.E.S.S. array (see Fig. 18), in order to lower the threshold of the expanded stereoscopic system and to prototype and test the capabilities of a significantly larger telescope (see Fig. 19).

Beyond these extensions of existing instruments, there are worldwide activities to build a fourth generation of arrays. In the European context, with Japanese participation, joint plans for a large array are in an advanced stage.

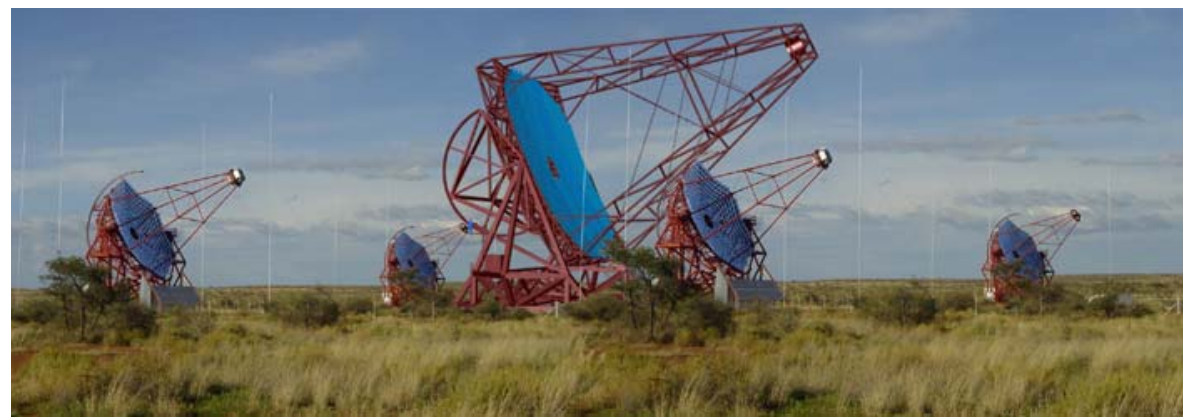

Fig. 18 Photomontage of H.E.S.S. Phase II (courtesy W. Hofmann). The $28 \mathrm{~m}$ telescope in the center of the original H.E.S.S. array should increase the sensitivity of the system by a factor $\approx 2$, at a threshold energy of $\sim 70 \mathrm{GeV}$ in coincidence mode 
Fig. 19 Build-up, in Namibia, of the dish and mirror support structure for the H.E.S.S. II telescope (courtesy M. Panter). First light is expected at the end of 2009

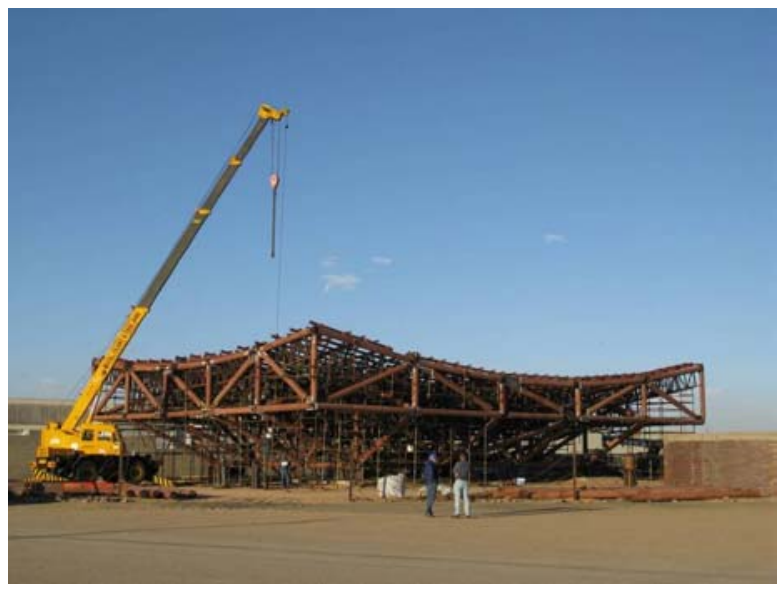

The project is called Cherenkov Telescope Array (CTA) [37] which should enhance the sensitivity at $1 \mathrm{TeV}$ by a further order of magnitude, down to the level of $10^{-3}$ of the flux from the Crab Nebula (1 mCrab). In the US the project Advanced Gamma-ray Imaging System (AGIS) [38] is actively pursued, with a similar goal.

The CTA project involves different types and sizes of telescopes (see Fig. 20) designed to extend the present energy range in both directions: to lower energies of some tens of $\mathrm{GeV}$ and to higher energies in excess of $100 \mathrm{TeV}$. Apart from deep broadband investigations of Galactic objects, this should also allow the investigation of the non-thermal aspects of large-scale structure formation in the Universe by studying active galactic nuclei, clusters of galaxies, and the related phenomena of accretion, starbursts, and galaxygalaxy mergers. These goals clearly require the coverage of a large energy range.

Fig. 20 Possible configuration of the CTA project, involving a mix of telescopes of various sizes and characteristics (courtesy W. Hofmann)

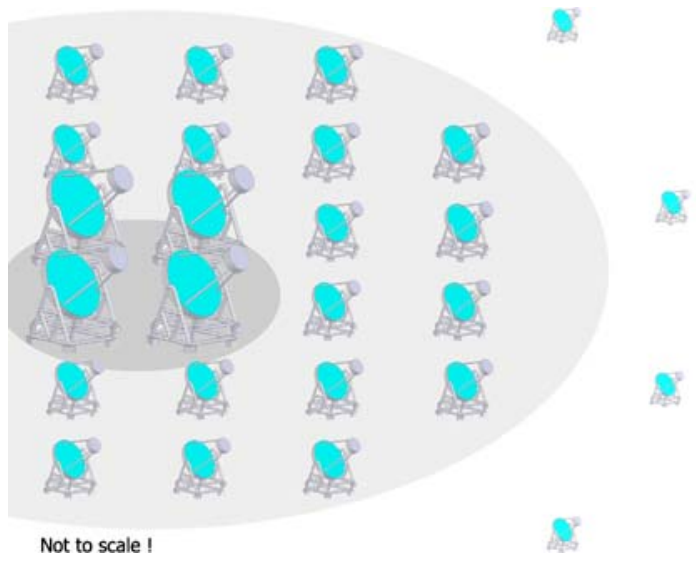


Acknowledgements We are indebted to our colleagues in the HEGRA and H.E.S.S. Collaborations, and especially in the Max-Planck-Institut für Kernphysik for many discussions over the years on the physics of $\gamma$-ray observations with imaging Cherenkov telescopes. We also thank W. Hofmann, G. Hermann and M. Panter for allowing us to use illustrative figures which they had designed for other purposes.

Open Access This article is distributed under the terms of the Creative Commons Attribution Noncommercial License which permits any noncommercial use, distribution, and reproduction in any medium, provided the original author(s) and source are credited.

\section{References}

1. H.E.S.S. Collaboration: http://www.mpi-hd.mpg.de/HESS/ (2009)

2. Bernöhr, K.: Simulations of imaging atmospheric Cherenkov telescopes with CORSIKA and sim_telarray. Astropart. Phys. 30, 149 (2008)

3. Heck, D. et al.: CORSIKA: a Monte Carlo code to simulate extensive air showers. Tech. report FZKA 6019. Forschungszentrum Karlsruhe (1998)

4. Galbraith, W., Jelly, J.V.: Light pulses from the night sky associated with Cosmic Rays. Nature 171, 351 (1953)

5. Nesterova, N.M., Chudakov, A.E.: Observation of Cherenkov radiation accompanying extensive air showers of cosmic rays. Sov. Phys. JETP 28, 385 (1955)

6. Zatsepin, V.I., Chudakov, A.E.: On the methods of searching for local sources of high energy photons. J. Exp. Theor. Phys. 41, 655 (1961)

7. Chudakov, A.E., Dadykin, V.L., Zatsepin, V.I., Nesterova, N.M.: A search for photons with energy $10^{13} \mathrm{eV}$ from local sources of cosmic radiation. Proc. P.N. Lebedev Phys. Inst. 26, 118 (1964) (in Russian); English Translation: Consultants Bureau 26, 99 (1965)

8. Lidvansky, A.S.: Air Cherenkov Methods in cosmic rays: a review and some history. Radiat. Phys. Chem. 75, 891 (2006) (archive:astro-ph/0504269)

9. Weekes, T.C.: The atmospheric Cherenkov technique in very high energy gamma-ray astronomy. SSRv 75, 1 (1996)

10. Weekes, T.C., et al. (Whipple Collaboration): Observation of TeV gamma rays from the Crab nebula using the atmospheric Cherenkov imaging technique. Aerosp. Power J. 342, 379 (1989)

11. Humensky, B.: University of Chicago. http://astro.uchicago.edu/cosmos/projects/veritasstereo/ (2004)

12. Hillas, A.M.: Cherenkov light images of EAS produced by primary gamma rays and by nuclei. Proc. 19th Int. Cosmic Ray Conf. (La Jolla) 3, 445 (1985)

13. Daum, A. (HEGRA Collaboration): First results on the performance of the HEGRA IACT array. Astropart. Phys. 8, 1 (1997)

14. HEGRA Collaboration and Max Planck Institut für Kernphysik: http://www.mpi-hd.mpg.de/ CT/ (1996)

15. Funk, S., et al.: The trigger system of the H.E.S.S. telescope array. Astropart. Phys. 22, 285 (2004)

16. Ong, R.A.: Very high-energy gamma-ray astronomy. Phys. Rep. 305, 93 (1998)

17. Weekes, T.C., et al.: VERITAS: the very energetic radiation imaging telescope array system. Astropart. Phys. 17, 221 (2003)

18. Mori, M., and the CANGAROO-III Team: Recent results form CANGAROO-II\&III. In: Aharonian, F.A., Völk, H.J., Horns, D. (eds.) High Energy Gamma-Ray Astronomy, AIP Proc., vol. 745, pp. 639ff. Melville, New York (2005)

19. Benbow, W. for the H.E.S.S. Collaboration: The status and performance of H.E.S.S.. In: Aharonian, F.A., Völk, H.J., Horns, D. (eds.) High Energy Gamma-Ray Astronomy, AIP Proc., vol. 745, pp.611 ff. Melville, New York (2005)

20. Lorenz, E. (for the MAGIC Collaboration): The MAGIC telescope, status and future plans. In: Aharonian, F.A., Völk, H.J., Horns, D. (eds.) High Energy Gamma-Ray Astronomy, AIP Proc., vol. 745, pp. 622ff. Melville, New York (2005) 
21. Aharonian, F.A., Hofmann, W., Konopelko, A.K., Völk, H.J.: The potential of ground based arrays of imaging atmospheric Cherenkov telescopes; I. Determination of shower parameters. Astropart. Phys. 6, 343 (1997)

22. Aharonian, F.A., Hofmann, W., Konopelko, A.K., Völk, H.J.: The potential of ground based arrays of imaging atmospheric Cherenkov telescopes; II. Gamma-ray flux sensitivities. Astropart. Phys. 6, 369 (1997)

23. CANGAROO Collaboration: http://icrhp9.icrr.u-tokyo.ac.jp/gallery.html (2004)

24. MAGIC Collaboration: http://wwwmagic.mppmu.mpg.de/ (2008)

25. Criswell, S., VERITAS Collaboration: http://veritas.adlerplanetarium.org/ (2007)

26. Drury, L.O'C., Aharonian, F.A., Völk, H.J.: The gamma-ray isibility of supernova remnants. A test of cosmic ray origin. A\&A 287, 959 (1994)

27. Völk, H.J., Aharonian, F.A., Breitschwerdt, D.: The nonthermal energy content and gammaray emission of starburst galaxies and clusters of galaxies. SSRv 75, 279 (1996)

28. Aharonian, F. et al. (H.E.S.S. Collaboration): Observations of the Crab Nebula with H.E.S.S. A\&A 457, 899 (2006)

29. Pfeffermann, E. \& Aschenbach, B.: In: Zimmermann, H., Trümper, J. (eds.) Roentgenstrahlung from the Universe. MPE Report 263, Garching:MPE, 267 (1996)

30. Uchiyama, Y., Takahshi, T., Aharonian, F.A.: Flat spectrum X-ray emission from the direction of a molecular cloud associated with SNR RX J1713.7-3946. PASJ 54, L73 (2002)

31. Aharonian, F. (H.E.S.S. Collaboration): Direct evidence for high-energy particle acceleration in the shell of a supernova remnant. Nature 432, 75 (2004)

32. Aharonian, F. (H.E.S.S. Collaboration): A detailed spectral and morphological study of the gamma-ray supernova remnant RX J1713.7-3946 with H.E.S.S.. A\&A 449, 223 (2006)

33. Aharonian, F. (H.E.S.S. Collaboration): Primary particle acceleration above $100 \mathrm{TeV}$ in the shell-type supernova remnant RX J1713.7-3946 with deep H.E.S.S.observations. A\&A 464, 235 (2007)

34. Berezhko, E.G., Völk, H.J.: Theory of cosmic ray production in the supernova remnant RX J1713.7-3946. A\&A 451, 981 (2006)

35. Berezhko, E.G., Völk, H.J.: Hadronic versus leptonic origin of the gamma-ray emission from Supernova Remnant RX J1713.6-3946. A\&A arXiv:0810.0988 [astro-ph] (2008)

36. H.E.S.S. Collaboration: Source of the month: Dec. 2007. http://www.mpi-hd.mpg.de/hfm/ HESS/pages/home/som/2007/12/ (2007)

37. Cherenkov Telescope Array: The Cherenkov Telescope Array (CTA) Project. http://www. cta-observatory.org/ (2009)

38. Buckley, J. et al.: The status and future of ground-based TeV gamma-ray astronomy. A white paper prepared for the Division of Astrophysics of the American Physical Society. arXiv:0810.0444 (2008) 